OPEN ACCESS

Edited by:

Marina I. Arleevskaya, Kazan State Medical Academy, Russia

Reviewed by: Alexei B. Shevelev, Russian Academy of

Sciences, Russia

Tatiana V. Kuznetsova, National Institute for Health

Development, Estonia

Lydia Bogomolnaya Texas A\&M University Health Science Center, United States

*Correspondence: Moffat Malishen malishenitasheni@gmail.com

Specialty section: This article was submitted to Microbial Immunology, a section of the journal Frontiers in Immunology

Received: 28 May 2017 Accepted: 07 July 2017 Published: 04 August 2017

Citation: Malisheni M, Khaiboullina SF Rizvanov AA, Takah N, Murewanhema $G$ and Bates $M$ (2017) Clinical Efficacy, Safety, and Immunogenicity of a Live Attenuated

Tetravalent Dengue Vaccine (CYD-TDV) in Children: A Systematic Review with Meta-analysis.

Front. Immunol. 8:863. doi: 10.3389/fimmu.2017.00863

\section{Clinical Efficacy, Safety, and Immunogenicity of a Live Attenuated Tetravalent Dengue Vaccine (CYD- TDV) in Children: A Systematic Review with Meta-analysis}

\author{
Moffat Malisheni ${ }^{1,2,3,4 *}$, Svetlana F. Khaiboullina ${ }^{5,6}$, Albert A. Rizvanov ${ }^{6}$, Noah Takah ${ }^{2,7}$, \\ Grant Murewanhema ${ }^{2,8}$ and Matthew Bates ${ }^{9,10}$ \\ ${ }^{1}$ Ministry of Health, Lusaka, Zambia, ${ }^{2}$ Institute of Health and Wellbeing, University of Glasgow, Glasgow, United Kingdom, \\ ${ }^{3}$ Department of Microbiology and Immunology, National University of Singapore, Singapore, Singapore, ${ }^{4}$ Institute of \\ Molecular and Cell Biology, Agency for Science, Technology and Research, Singapore, Singapore, ${ }^{5}$ Department of \\ Microbiology and Immunology, University of Nevada Reno, Reno, NV, United States, ${ }^{6}$ Kazan Federal University, Kazan, \\ Russia, ${ }^{7}$ Ministry of Health, Yaounde, Cameroon, ${ }^{8}$ College of Health Sciences, University of Zimbabwe, Harare, Zimbabwe, \\ ${ }^{9}$ University College London Research \& Training Programme, University of Zambia, University Teaching Hospital, Lusaka, \\ Zambia, ${ }^{10}$ HerpeZ, University Teaching Hospital, Lusaka, Zambia
}

Background: Dengue hemorrhagic fever is the leading cause of hospitalization and death in children living in Asia and Latin America. There is an urgent need for an effective and safe dengue vaccine to reduce morbidity and mortality in this high-risk population given the lack of dengue specific treatment at present. This review aims to determine the efficacy, safety, and immunogenicity of CYD-TDV vaccine in children.

Methods: This is a systematic review including meta-analysis of randomized controlled clinical trial data from Embase, Medline, the Cochrane Library, Web of Science, and ClinicalTrials.gov. Studies that assessed CYD-TDV vaccine efficacy [(1 - RR)*100], safety (RR), and immunogenicity (weighted mean difference) in children were included in this study. Random effects model was employed to analyze patient-level data extracted from primary studies.

Results: The overall efficacy of CYD-TDV vaccine was 54\% (40-64), while serotypespecific efficacy was 77\% (66-85) for DENV4, 75\% (65-82) for DENV3, 50\% (36-61) for DENV1, and 34\% (14-49) for DENV2. 15\% (-174-74) vaccine efficacy was obtained for the unknown serotype. Meta-analysis of included studies with longer follow-up time (25 months) revealed that CYD-TDV vaccine significantly increased the risk of injection site reactions $(R R=1.1: 1.04-1.17 ; p$-value $=0.001)$. Immunogenicity (expressed as geometric mean titers) in descending order was 439.7 (331.7-547.7), 323 (247 - 398.7), 144.1 (117.9-170.2), and 105 (88.7-122.8) for DENV3, DENV2, DENV1, and DENV4, respectively.

Conclusion: CYD-TDV vaccine is effective and immunogenic in children overall. Reduced efficacy of CYD-TDV vaccine against DENV2 notoriously known for causing severe dengue infection and dengue outbreaks cause for serious concern. Post hoc 
meta-analysis of long-term follow-up data ( $\geq 25$ months) from children previously vaccinated with CYD-TDV vaccine is needed to make a conclusion regarding CYD-TDV vaccine safety in children. However, CYD-TDV vaccine should be considered for use in regions where DENV2 is not endemic as currently there is no specific treatment for dengue infection.

Keywords: dengue hemorrhagic fever, dengue shock syndrome, CYD-TDV, dengue virus, efficacy, safety, immunogenicity

\section{INTRODUCTION}

Continuously increasing dengue virus (DENV) related morbidity and mortality poses a serious threat to global public health and has exerted pressure on national health budgets of endemic countries. There are four types of genetically distinct dengue viruses (DENV 1-4) (1), all causing severe dengue infection $(2,3)$. Brady et al. estimates that four billion people are at risk of acquiring dengue infection worldwide (4) with approximately 284-528 million dengue cases being documented annually (5). Dengue hemorrhagic fever (DHF)/Dengue Shock Syndrome (DSS) comprise 500,000 to one million of these cases leading to over 20,000 fatalities mostly in children $(6,7)$. The goal of World Health Organization is to reduce dengue related morbidity and mortality by 2020 (8). Despite the availability of vector control programs, dengue infection has continued to rise globally (9) with significant economic burdens and might continue to do so in the future given the ongoing climate change. Introducing an efficacious and safe vaccine in endemic regions has the potential to reduce dengue related hospitalization and death in children due to severe dengue infection (DHF/DSS). The risk of developing DHF/ DSS during secondary infection is increased when an individual is exposed to a dengue serotype that is different from the one previously experienced (10). This occurs due to antibody-dependent enhancement (ADE) which involves low levels of cross-reactive neutralizing antibodies produced during primary dengue infection forming complexes with target cell receptors $(3,11,12)$. Consequently enhancing the number of dengue-infected cells and viremia $(3,11,12)$. Therefore, a tetravalent dengue vaccine capable of eliciting a balanced immune response against all four dengue serotypes is warranted if complications due to $\mathrm{ADE}$ are to be averted $(11,13,14)$. CYD-TDV vaccine is the most advanced live attenuated tetravalent dengue vaccine (15). However, comprehensive evidence regarding CYD-TDV vaccine efficacy, safety, and immunogenicity in children exclusively is absent.

Abbreviations: ADE, antibody-dependent enhancement; AE, adverse events; AR, adverse reactions; CYD-TDV, live-tetravalent dengue vaccine; DENV, dengue virus; DHF, dengue hemorrhagic fever; DSS, dengue shock syndrome; ELISA, enzyme-linked immunosorbent assay; GMT, geometric mean titre; JE, Japanese encephalitis; PRISMA, preferred reporting items for systematic reviews and meta-analyses; PRNT50, plaque reduction neutralisation test with a 50\% plaque reduction threshold; RCT, randomized controlled trial; RR, relative risk; RT-PCR, reverse transcription polymerase chain reaction; SAE, severe adverse events; UAE, unsolicited adverse events; UN, United Nations; VCD, virologically confirmed dengue; WHO, World Health Organization; WMD, weighted mean difference; YF, yellow fever.
A meta-analysis of randomized controlled trials (RCTs) by da Costa et al. has demonstrated that CYD-TDV vaccine is safe and induces a balanced immune response (16). However, safety and immunogenicity were determined for all age groups and no subgroup analysis based on the age of included participants was conducted. The fact that dengue infection is more severe in children compared to adults indicates that the two groups might respond to CYD-TDV vaccine differently with a possibility of adults confounding the true effect of the vaccine in children. The study also assessed vaccine efficacy by combining five primary studies. However, only Sabchareon et al. out of the five combined studies was designed to determine CYD-TDV vaccine efficacy in Thai children (17). The determined vaccine efficacy was $30.2 \%$ and was not statistically significant. Two large Phase III clinical studies designed to determine CYD-TDV vaccine efficacy and not included in the meta-analysis by da Costa et al. have been conducted in Asian and Latin American children showing efficacy of 56.5 and $60.8 \%$, respectively $(9,18)$. However, because these studies were conducted in various age groups from different regions, the findings are not directly comparable. Therefore, to comprehensively address all the aforementioned concerns, we decided to determine the efficacy, safety, and immunogenicity of CYD-TDV vaccine in children by answering the following questions: (i) does CYD-TDV vaccine reduce the incidence of virologically confirmed dengue (VCD) cases in vaccinated compared with unvaccinated children? (ii) Does CYD-TDV vaccine increase the risk of adverse events in vaccinated as compared to the unvaccinated children? (iii) Is there a difference in geometric mean titers (GMTs) between children exposed to CYD-TDV vaccine and those unexposed?

\section{METHODS}

\section{Eligibility Criteria and Definitions}

This review was conducted and reported in accordance with the Cochrane and preferred reporting items for systematic reviews and meta-analyses guidelines (19, 20). Population: children were defined as all individuals under the age of 18 years (21). Intervention: CYD-TDV vaccine manufactured by Sanofi Pasteur. Vaccine was reconstituted in $0.4 \%$ sodium chloride and $2.5 \%$ serum albumin. Comparator: standard of care, placebo, or no intervention. Outcome: the primary end assessing points were CYD-TDV vaccine efficacy in accordance with the "Guidelines for clinical trials of dengue vaccine in endemic areas" (22). Reduction in the incidence of VCD cases per protocol analysis. Safety: AEs-unfavorable medical occurrences that are not treatment related and ARs-those that might be treatment related 
(23). Immunogenicity: levels of dengue neutralizing antibodies expressed as GMTs and measured using plaque reduction neutralisation test with a 50\% plaque reduction threshold (PRNT50). More information on the PRNT50 test can be obtained in the "guidelines for plaque reduction neutralization testing of human antibodies to dengue viruses" (24). Study design: only RCTs were included in this review. CYD-TDV vaccination interval requirement was that immunizations be conducted at months 0,6 , and 12 (three vaccine regimen). Exclusion criteria: studies which did not assess CYD-TDV vaccine efficacy, safety, or immunogenicity or did not use CYD-TDV vaccine; studies involving participants aged over 17 years; and studies that used non-RCT study designs, non-three vaccine regimen or a three vaccine regimen with a different vaccination interval.

\section{Literature Search and Data Extraction}

A comprehensive search strategy was developed in collaboration with an experienced medical librarian to identify recently published studies as presented in Appendix I (all referenced appendices are in the Supplementary Material). Embase, Medline, Web of Science, the Cochrane Library, ClinicalTrials. gov, references of included studies, and authors served as sources for published data. Gray literature was not searched because it lacks quality control. The search for published data was initiated on $01 / 03 / 2016$, concluded on 11/05/2016 and pilot tested according to the method proposed by Long (25) (Appendix II, Figure 1 in Supplementary Material). This was done to ensure that the analysis includes all relevant information and fit to achieve the goal of this study. Corresponding authors of primary studies were contacted to request for numerical data or clarifications in cases where data were incomplete or graphically presented (Appendix II, Figure 2 in Supplementary Material). Information was extracted based on individual patient-level data.

\section{Data Items and Summary Measures}

Primary end points and their respective summary measures included: overall and serotype-specific CYD-TDV vaccine efficacy (per protocol analysis), CYD-TDV safety [immediate AEs, severe adverse events (SAEs), solicited ARs, solicited injection site reactions (pain, erythema, and swelling), solicited systemic reactions (fever, headache, malaise, myalgia, and asthenia) and unsolicited adverse event (UAEs)]. Relative risk (RR) was the preferred summary measure for CYD-TDV efficacy $\left[(1-R R)^{\star} 100\right]$ (17) and safety, whereas immunogenicity (measured as GMTs) was estimated using the weighted mean difference (WMD). Relative risk was defined as the ratio of incidence VCD cases in the CYD-TDV vaccine group divided by the ratio of incidence VCD cases in the unvaccinated group (26). Mean difference was defined as an absolute difference between the GMTs in the intervention and control groups.

\section{Risk of Bias and Statistical Analysis}

The Cochrane Handbook for Systematic Reviews of Interventions tool (27) was used in this analysis to assess the quality of each of the included studies. The risk of bias was assessed both at the study and the outcome levels. Evidence tables served as the starting point for data synthesis. The tables were reviewed as well as the forest plots to determine the possibility of combining data from studies in a meta-analysis. The $I^{2}$ and $Q$ statistics were used to formally check for the presence of heterogeneity and consequently determine whether the effect sizes should be pooled. Heterogeneity was classified as low, medium and high for $I^{2}$ values corresponding to 25, 50, and $75 \%$, respectively (28). If heterogeneity was either low/medium or reduced to these levels after being resolved, the pooled effect sizes of outcomes were explored. However, if variations in the effect size between pooled studies remained high $\left(I^{2} \geq 75 \%\right)$ after efforts to resolve heterogeneity, meta-analysis was not conducted. The heterogeneity was investigated using metaregression and subgroup analysis to explain its possible cause. The Cochrane Collaboration recommends that studies with divergent effect sizes from the rest should be excluded to resolve heterogeneity (27). The influence of individual studies on the overall effect size was formally investigated using meta-influence. Identified studies were removed systematically to reduce heterogeneity across the combined studies. Sensitivity analysis was performed to explore the robustness of the findings using the fixed effects model (Appendices III and IV in Supplementary Material). The random effects model was preferred because the true effect size may not be constant across all the included studies (29) given that they were conducted in different age groups, countries, regions, and ethnic groups. Extracted data were exported from Excel spreadsheet to STATA version 13.0, where all statistical analyses were conducted. Publication bias was explored by employing Harbord's and Egger's tests.

\section{RESULTS}

336 articles were selected for this study. Embase, Medline, Web of Science, the Cochrane Library, and ClinicalTrials.gov yielded 104, 80, 53, 46, and 53 articles, respectively (Figure 1 below). Duplicate studies were removed using Mendeley reference manager leaving 194 published articles for further analysis. Of these, 174 articles were removed based on titles and abstracts. After an in-depth review, additional eight articles were excluded, including a press release (30), an abstract (31), one paper used a two regimen vaccination protocol (32), two papers used different vaccines, Acambis (33) and PVRV (34), and one meta-analysis of RCTs previously mentioned (16). Also, two studies utilizing a different protocol of the vaccination regimen and vaccine reconstitution protocol were excluded $(35,36)$. At the end of the selection procedure, nine studies were found eligible for inclusion in meta-analysis (9, $17,18,37-42$ ) after one study was excluded (43) because it presented all data graphically.

A summary of the included studies' characteristics is presented in Table 1. Additional study activities are presented in Appendix II, Table 1 in Supplementary Material. All three studies that assessed vaccine efficacy used reverse transcription polymerase chain reaction and enzyme-linked immunosorbent assay tests to confirm dengue cases. Six studies did not specify how information regarding CYD-TDV vaccine safety was collected, while four requested parents/guardians of participants to record safety profiles. PRNT50 was utilized 


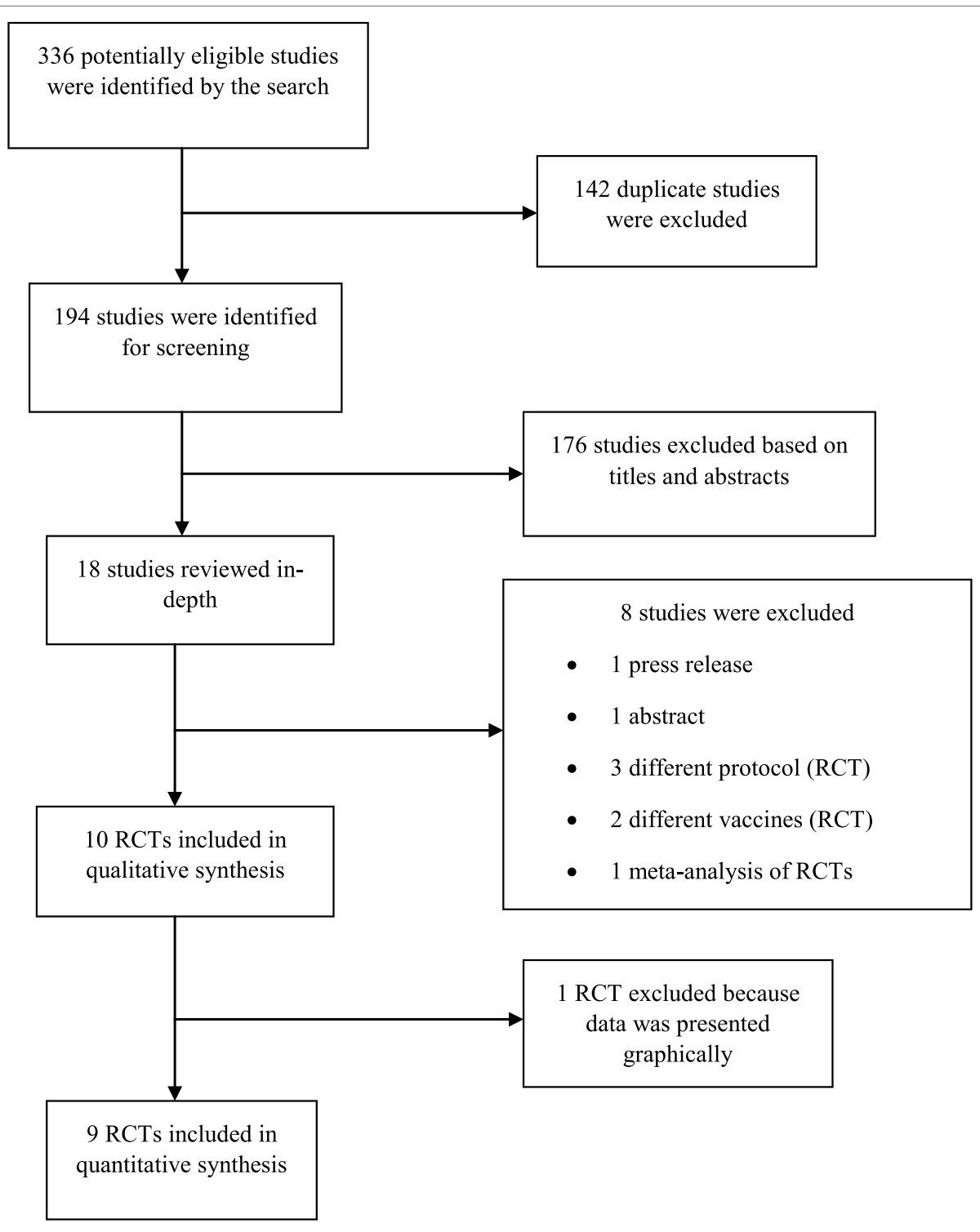

FIGURE 1 | Study selection process.

by all included studies to determine immunogenicity. Details regarding the aforesaid are summarized in Appendix II, Table 2 in Supplementary Material. Quality assessment of eligible studies is presented in Table 2. Regarding RR and WMD analyses the values of no difference were 1 and 0 , respectively. Therefore, 95\% confidence intervals $(95 \% \mathrm{CI})$ that traversed 1 regarding the former and 0 the latter were considered statistically insignificant (alpha $>0.05$ ). This review considered three hypotheses: CYD-TDV vaccination does not reduce the incidence of VCD cases in children; CYD-TDV vaccination increases the risk of ARs in children; and there is no difference in GMT levels between vaccinated and unvaccinated children. Variables used in meta-regression and subgroup analyses included: gender, sample size, randomization ratio, blinding method, placebo type, age group, study location, RCT phase, and flavivirus (DENV, yellow fever, and Japanese encephalitis) seroprevalence at baseline.

\section{CYD-TDV Vaccine Efficacy}

The review found a statistically significant pooled overall CYDTDV vaccine efficacy of $54 \%(40-64$; $p$-value $<0.001)$. This result implies that the vaccine reduces the risk of acquiring dengue infection in the intervention group relative to the control group by $54 \%$. Serotype-specific efficacy showed that CYD-TDV vaccine was more effective against DENV4 (77\%: 66-85; $p$-value $<0.001)$ and DENV3 (75\%: 65-82; $p$-value $<0.001)$, while it was less effective against DENV1 (50\%: 36-61; $p$-value $<0.001$ ), DENV2 (34\%: $14-49 ; p$-value $=0.002)$ and unknown DENV serotype $(15 \%:-174-74 ; p$-value $=0.79)$. There was convincing evidence to reject the pre-specified null hypothesis for all but the unknown 
TABLE 1 | Study characteristics of the vaccine trials that meet the inclusion criteria.

\begin{tabular}{|c|c|c|c|c|c|c|c|c|c|}
\hline \multirow[t]{2}{*}{ Reference } & \multirow{2}{*}{$\begin{array}{l}\text { Study } \\
\text { design } \\
\text { (phase) }\end{array}$} & \multicolumn{2}{|c|}{ Sample size } & \multirow{2}{*}{$\begin{array}{l}\text { Age range } \\
\text { (years) }\end{array}$} & \multicolumn{2}{|c|}{ Males } & \multirow[t]{2}{*}{ Outcome determined } & \multicolumn{2}{|c|}{ Seroprevalence at baseline } \\
\hline & & CYD-TDV & Placebo & & $\begin{array}{l}\text { CYD-TDV } \\
(\%)\end{array}$ & $\begin{array}{l}\text { Placebo } \\
(\%)\end{array}$ & & CYD-TDV & Placebo \\
\hline $\begin{array}{l}\text { Crevat } \\
\text { et al. (43) }\end{array}$ & $\mathrm{RCT}(I I)$ & 60 & 30 & $1-1.25$ & 63 & 60 & Safety and immunogenicity & DENV or JE; $45 \%$ & DENV or JE; $50 \%$ \\
\hline $\begin{array}{l}\text { Villar } \\
\text { et al. (9) }\end{array}$ & $\mathrm{RCT}(\mathrm{III})$ & 12,574 & 6,261 & $9-16$ & 49.7 & 49.6 & Efficacy and safety & DENV; $80 \%$ & DENV; 77\% \\
\hline $\begin{array}{l}\text { Capeding } \\
\text { et al. (18) }\end{array}$ & $\mathrm{RCT}(\mathrm{III})$ & 6,851 & 3,424 & $2-14$ & 48 & 48 & Efficacy and safety & DENV or JE; $79 \%$ & DENV or JE; $77 \%$ \\
\hline $\begin{array}{l}\text { Amar-Singh } \\
\text { et al. (40) }\end{array}$ & $\mathrm{RCT}(\mathrm{III})$ & 199 & 51 & $2-11$ & 48 & 62.7 & Safety and immunogenicity & $\begin{array}{l}\text { FV-positive; 44\% } \\
\text { FV-negative; 35\% }\end{array}$ & \\
\hline $\begin{array}{l}\text { Villar } \\
\text { et al. (41) }\end{array}$ & $\operatorname{RCT}(I I)$ & 401 & 199 & $9-16$ & 49.1 & 45.7 & Safety and immunogenicity & $\mathrm{FV} ; 78.8 \%$ & FV; $80.8 \%$ \\
\hline $\begin{array}{l}\text { Dayan } \\
\text { et al. (37) }\end{array}$ & $\mathrm{RCT}(I I)$ & 100 & 50 & $9-16$ & 40 & 55 & Safety and immunogenicity & DENV or YF; $81 \%$ & DENV or YF; 84\% \\
\hline $\begin{array}{l}\text { Leo } \\
\text { et al. (38) }\end{array}$ & $\mathrm{RCT}(\mathrm{II})$ & 898 & 300 & $2-17$ & 44.0 & 54.4 & Safety and immunogenicity & Not stated & Not stated \\
\hline $\begin{array}{l}\text { Tran } \\
\text { et al. (39) }\end{array}$ & $\mathrm{RCT}(\mathrm{II})$ & 120 & 60 & $2-17$ & 48 & 58 & Safety and immunogenicity & DENV or JE; $71 \%$ & DENV or JE; $78 \%$ \\
\hline $\begin{array}{l}\text { Sabchareon } \\
\text { et al. (17) }\end{array}$ & $\mathrm{RCT}(\mathrm{llb})$ & 2,669 & 1,333 & $4-11$ & 48 & 48 & $\begin{array}{l}\text { Efficacy, safety, and } \\
\text { immunogenicity }\end{array}$ & DENV or JE; $91 \%$ & DENV or JE; $92 \%$ \\
\hline $\begin{array}{l}\text { Lanata } \\
\text { et al. (42) }\end{array}$ & RCT (II) & 199 & 99 & $2-11$ & 51 & 46 & Safety and immunogenicity & DENV; 37\% & DENV; 48\% \\
\hline
\end{tabular}

RCT, randomized controlled trial; DENV, dengue virus; YF, yellow fever; JE, Japanese encephalitis; FV, flavivirus.

TABLE 2 | Risk of bias assessment.

\begin{tabular}{|c|c|c|c|c|c|c|c|c|}
\hline \multirow[t]{2}{*}{ Reference } & \multicolumn{2}{|c|}{ Selection bias } & \multicolumn{2}{|c|}{ Blinding } & \multirow[t]{2}{*}{ Attrition } & \multirow[t]{2}{*}{ Reporting bias } & \multirow[t]{2}{*}{ Other bias } & \multirow[t]{2}{*}{ Researcher's judgment } \\
\hline & Randomization & Concealment & Performance & Detection & & & & \\
\hline Crevat et al. (43) & Low & Unknown & Low & Unknown & Low & Low & Unknown & Unknown \\
\hline Villar et al. (9) & Low & Low & Low & Low & Low & Low & Unknown & Low \\
\hline Capeding et al. (18) & Low & Low & Low & Low & Low & Low & Unknown & Low \\
\hline Amar-Singh et al. (40) & High & Low & High & Low & Low & Low & Unknown & High \\
\hline Villar et al. (41) & Low & Low & High & Low & Low & Low & Unknown & High \\
\hline Dayan et al. (37) & Low & Low & High & Low & Low & Low & Unknown & High \\
\hline Leo et al. (38) & Low & Low & High & Low & Low & Low & Unknown & High \\
\hline Tran et al. (39) & Low & Low & High & Low & Low & Low & Unknown & High \\
\hline Sabchareon et al. (17) & Low & Low & High & Low & Low & Low & Unknown & High \\
\hline Lanata et al. (42) & Low & Low & Low & Low & Low & Low & Unknown & Low \\
\hline Risk of bias & \multicolumn{2}{|l|}{ Interpretation } & \multicolumn{3}{|l|}{ Within trial bias } & \multicolumn{3}{|l|}{ Across trial bias } \\
\hline Low & \multicolumn{2}{|c|}{$\begin{array}{l}\text { Bias, if present, is unlikely to alter } \\
\text { the results seriously }\end{array}$} & \multicolumn{3}{|c|}{ Low risk of bias for all key domains } & \multicolumn{3}{|c|}{ Most information is from trials at low risk of bias } \\
\hline Unknown & \multicolumn{2}{|c|}{$\begin{array}{l}\text { A risk of bias that raises some } \\
\text { doubt about the results }\end{array}$} & \multicolumn{3}{|c|}{$\begin{array}{l}\text { Low or unclear risk of bias for all key } \\
\text { domains }\end{array}$} & \multicolumn{3}{|c|}{ Most information is from trials at low or unclear risk of bias } \\
\hline High & \multicolumn{2}{|c|}{ Bias may alter the results seriously } & \multicolumn{3}{|c|}{$\begin{array}{l}\text { High risk of bias for one or more key } \\
\text { domains }\end{array}$} & \multicolumn{3}{|c|}{$\begin{array}{l}\text { The proportion of information from trials at high risk of } \\
\text { bias is sufficient to affect the interpretation of results }\end{array}$} \\
\hline
\end{tabular}

serotype, which was not statistically significant. The main findings for CYD-TDV efficacy including evidence for the presence of heterogeneity and publication bias are summarized in Table 3.

\section{CYD-TDV Vaccine Safety}

Generally, solicited injection site reactions (any) and solicited systemic reactions (any, fever, headache, and asthenia) showed an increased but statistically insignificant risk in vaccinated children compared with unvaccinated children. Other than the aforesaid, CYD-TDV vaccine reduced the risk of ARs. However, meta-analysis of included studies with longer follow-up time (25 months) revealed that CYD-TDV vaccination increased the risk of solicited injection site reactions; $\mathrm{RR}=1.1(1.04-1.17$; $p$-value $=0.001)$ and $\mathrm{RR}=1.09(0.97-1.22 ; p$-value $=0.145)$ using the fixed and random effects models, respectively. However, only the fixed model showed a statistically significant risk (Appendix 
TABLE 3 | Main CYD-TDV efficacy findings.

\begin{tabular}{|c|c|c|c|c|c|c|c|c|}
\hline $\begin{array}{l}\text { CYD-TDV } \\
\text { efficacy }\end{array}$ & $\begin{array}{c}\text { Number of } \\
\text { studies pooled }\end{array}$ & $\begin{array}{l}\text { Intervention } \\
n / N\end{array}$ & $\begin{array}{l}\text { Control } \\
n / N\end{array}$ & $\begin{array}{l}\text { Heterogeneity } \\
\text { (p-value) }\end{array}$ & $\begin{array}{l}\text { Harbord's test } \\
\text { for publication } \\
\text { bias ( } p \text {-value) }\end{array}$ & RR $(95 \% \mathrm{Cl})$ & $\begin{array}{c}\text { Efficacy }=(R R-1)^{\star} 100 \\
(95 \% \mathrm{Cl})\end{array}$ & $p$-Value \\
\hline Overall & 3 & $338 / 21,736$ & $386 / 10,832$ & $61.6 \%(0.074)$ & 0.396 & $0.46(0.36-0.60)$ & $54 \%(40-64)$ & $<0.001$ \\
\hline DENV1 & 3 & $126 / 21,736$ & $126 / 10,832$ & $0.0 \%(0.97)$ & 0.482 & $0.50(0.39-0.64)$ & $50 \%(36-61)$ & $<0.001$ \\
\hline DENV2 & 3 & $127 / 21,736$ & $96 / 10,832$ & $0.0 \%(0.45)$ & 0.359 & $0.66(0.51-0.86)$ & $34 \%(14-49)$ & 0.002 \\
\hline DENV3 & 3 & $54 / 21,736$ & 107/10,832 & $0.0 \%(0.91)$ & 0.062 & $0.25(0.18-0.35)$ & $75 \%(65-82)$ & $<0.001$ \\
\hline DENV4 & 3 & $35 / 21,736$ & 78/10,832 & $0.0 \%(0.61)$ & NA & $0.23(0.15-0.34)$ & $77 \%(66-85)$ & $<0.001$ \\
\hline $\begin{array}{l}\text { Unknown } \\
\text { serotype }\end{array}$ & 3 & $12 / 21,736$ & 6/10,832 & $17.3 \%(0.3)$ & NA & $0.85(0.26-2.74)$ & $15 \%(-174-74)$ & 0.79 \\
\hline
\end{tabular}

$N$, sample size; $n$, number of cases recorded; NA, not applicable (could not be calculated because input data contained 0 values); DENV 1-4, dengue virus serotypes.

TABLE 4 | Main CYD-TDV safety findings.

\begin{tabular}{|c|c|c|c|c|c|c|c|}
\hline Safety profile & $\begin{array}{l}\text { Number of studies } \\
\text { combined }\end{array}$ & $\begin{array}{l}\text { Intervention } \\
n / N\end{array}$ & $\begin{array}{l}\text { Control } \\
n / N\end{array}$ & $\begin{array}{l}\text { Heterogeneity } \\
\text { (p-value) }\end{array}$ & $\begin{array}{l}\text { Harbord's test for publication } \\
\text { bias ( } p \text {-value) }\end{array}$ & RR (95\% Cl) & $p$-Value \\
\hline SAE & 7 & $783 / 24,304$ & $447 / 12,020$ & $0.0 \%(0.51)$ & 0.52 & $0.86(0.77-0.96)$ & 0.009 \\
\hline Solicited reactions ${ }^{a}$ & 2 & $133 / 299$ & $110 / 149$ & $0.0 \%(0.41)$ & NA & $0.64(0.55-0.74)$ & $<0.001$ \\
\hline UAE & 7 & 1,709/4,239 & $847 / 2,003$ & $18.7 \%(0.29)$ & 0.02 & $0.95(0.88-1.03)$ & 0.19 \\
\hline \multicolumn{8}{|c|}{ Solicited injection site reactions occurring between day 0 and 7 post vaccination } \\
\hline Any $^{a}$ & 5 & $2,112 / 3,935$ & $898 / 1,848$ & $61.4 \%(0.035)$ & 0.64 & $1.06(0.97-1.16)$ & 0.2 \\
\hline Pain (any) ${ }^{a}$ & 3 & $151 / 663$ & $191 / 329$ & $52.2 \%(0.12)$ & 0.48 & $0.40(0.30-0.51)$ & $<0.001$ \\
\hline Erythema (any) & 5 & $72 / 1,140$ & $50 / 504$ & $67.1 \%(0.016)$ & 0.42 & $0.55(0.27-1.11)$ & 0.09 \\
\hline Swelling (any) & 5 & $50 / 1,140$ & $41 / 504$ & $67.2 \%(0.016)$ & 0.37 & $0.47(0.20-1.06)$ & 0.07 \\
\hline \multicolumn{8}{|c|}{ Solicited systemic reaction occurring between day 0 and 14 post vaccination } \\
\hline Any & 7 & $2,694 / 4,234$ & $1,266 / 1,998$ & $33.9 \%(0.17)$ & 0.52 & $1.0(0.95-1.06)$ & 0.9 \\
\hline Fever (any) & 5 & $187 / 1,138$ & $75 / 504$ & $48.1 \%(0.1)$ & 0.94 & $1.08(0.76-1.53)$ & 0.67 \\
\hline Headache (any) & 5 & $401 / 1,140$ & $173 / 504$ & $60.8 \%(0.03)$ & 0.44 & $1.0(0.78-1.3)$ & 0.97 \\
\hline Malaise (any) & 5 & $314 / 1,140$ & $133 / 504$ & $55.9 \%(0.059)$ & 0.96 & $0.99(0.75-1.32)$ & 0.96 \\
\hline Myalgia (any) & 5 & $312 / 1,140$ & $158 / 504$ & $68.0 \%(0.014)$ & 0.788 & $0.8(0.59-1.08)$ & 0.15 \\
\hline Asthenia (any) & 5 & $177 / 1,140$ & $69 / 504$ & $56.8 \%(0.055)$ & 0.093 & $1.14(0.75-1.74)$ & 0.55 \\
\hline
\end{tabular}

$N$, sample size; $n$, number of cases recorded; NA, not applicable (could not be calculated because few studies were pooled); SAE, severe adverse events; UAE, unsolicited adverse events.

${ }^{a}$ All parameters were determined after resolving heterogeneity by excluding divergent studies.

IV, Figure 8 in Supplementary Material). Insignificantly increased risk of solicited systemic reactions was also observed using both the random and fixed effects models (Appendix IV, Figures 10 and 11 in Supplementary Material). None of the aforementioned variables used in subgroup analyses were associated with finding of a statistically significant heterogeneity $\left(I^{2}>90 \%\right.$; $p$-value $\left.<0.001\right)$. However, meta-regression analysis demonstrated that gender explained $95.1 \%(p$-value $=0.049)$ and $96.5 \%(p$-value $=0.002)$ of the variability in the studies that assessed solicited reactions and solicited injection site reactions, respectively (Appendix IV, Figures 2 and 3 in Supplementary Material). Negative gender coefficients entailed that for every unit increase in the proportion of males in the CYD-TDV group, the log RR reduced by 0.45 and 0.91 units for solicited reactions and injection site reactions, respectively. Following stratification of solicited reactions and injection site reactions by gender, heterogeneity in the subgroups of the pooled studies with more males plummeted to $0.0 \%(p$-value $<0.41)$ and $61.4 \%(p$-value $=0.035)$, respectively (Table 4). A study by Crevat et al. found that the most frequently reported safety profiles in the intervention group were UAEs (60-70\%), solicited ARs (50-55\%), solicited systemic reactions
(40-50\%), and solicited injection site reactions (<20\%) (43). There were no immediate AEs or SAEs reported.

\section{CYD-TDV Vaccine Induced Immunogenicity}

Although it was challenging to determine the overall immunogenicity using the random effects model due to the persistence of variation in the effect sizes even after resolving serotype-specific heterogeneity, the fixed effects model generated $74.28 \mathrm{1} / \mathrm{dil}$ (69.90-78.68; $p$-value $<0.001)$. The combined serotype-specific GMT levels found after resolving heterogeneity in descending order was: DENV3 (439.7 1/dil), DENV2 (323 1/dil), DENV1 (144.1 1/dil), and DENV4 (105.71 1/dil). A different order was detected when the fixed effects model was applied; DENV3 (114.56 1/dil), DENV4 (112.34 1/dil), DENV2 (81.91 1/dil), and DENV1 (40.51 1/dil) (Appendix IV in Supplementary Material). This showed that the estimates of immunogenicity were not robust. Subgroup analysis and the systematic elimination of studies with divergent mean differences revealed that combining studies with different ages resulted in significant heterogeneity, except for DENV1. This was observed even within the same 
study (Appendix IV, Figures 4 and 5 in Supplementary Material). By contrast, pooling together studies with the same age resulted in low heterogeneity $\left(I^{2}=0.0 \%\right.$ ), except for DENV4 (Appendix IV, Figures 6 and 7 in Supplementary Material). The main immunogenicity findings and respective $95 \%$ CIs are summarized in Table 5. The highest neutralizing antibody titers in descending order reported by Cravat et al. were as follows: DENV3 (311-387 1/dil), DENV2 (147-213 1/dil), DENV4 (127-160 1/dil), and DENV1 (105-124 1/dil) (43). Tran et al. reported the following GMTs: DENV1-4:129, 216, 169, and 146 1/dil, respectively (39). The study further demonstrated that GMTs in children increased with increasing age: 2-5 years (64.7-143 1/dil), 6-11 years (93.9-185 1/dil) and 12-17 years (135-334 1/dil).

\section{DISCUSSION}

\section{CYD-TDV Vaccine Efficacy}

Although our findings show that CYD-TDV vaccine is protective against dengue infection overall, its reduced efficacy against DENV2 is extremely worrying. This is because DENV2 is known to cause severe dengue infection and is twice as likely to result in DHF/DSS compared to other serotypes (6). The Asian DENV2 has also been reported to cause outbreaks of DHF/DSS, highly pathogenic and is gradually replacing the less pathogenic Latin American variant (44). All efficacy studies conducted in Asia demonstrated reduced and statistically insignificant vaccine efficacy against DENV2 compared with the one conducted in Latin America. This might indicate that CYD-TDV vaccine induced antibodies readily neutralize the less pathogenic Latin American than the highly virulent Asian DENV2 variant. It has been reported that DENV2 neutralizing antibodies induced after primary infection with DENV1 demonstrate differential neutralizing activity against the Asian and Latin American DENV2 variants (45).

It is worth noting that the observed 32\% CYD-TDV vaccine protection against DENV2 is merely the best estimate. The real vaccine efficacy in the population can be as low as $14 \%$ (Table 3). Another important point to note is that CYD-TDV vaccine induced DENV2 neutralizing antibodies had the second highest GMT levels and yet provided the least protection. The question is why? Villar et al. concluded that GMT levels elicited by CYDTDV vaccine do not reflect serotype-specific vaccine efficacy (9).
It has also been suggested that dengue antibodies either might not be the immunological correlate of protection or that each dengue serotype has its own protective titer threshold (46). Both findings correspond with our results which show that CYD-TDV vaccine protection was highest for the serotype with the lowest GMT levels and so on (Tables 3 and 5). Microevolution due to genetic recombination and natural selection occurring within individual dengue serotypes might explain lower efficacy of the CYD-TDV vaccine against DENV2 (47-49). Wahala et al. have shown that mutations occurring in the E protein (the major target for dengue neutralizing antibodies) have an effect on antibody binding and neutralizing activity (50). Therefore, reduced vaccine efficacy against DENV2 could be due to the fact that the circulating DENV2 virus acquired mutations in the $\mathrm{E}$ protein hence becoming antigenically and genetically different from the one included in the CYD-TDV vaccine. Reduced DENV2 vaccine efficacy might as well be as a result of dominant CYD-TDV vaccine induced antibodies that lack or have low serotype-specific neutralizing activity against the circulating DENV2 serotype. This is because high levels of serotype-specific neutralizing antibodies are known to confer protection against subsequent DENV infection (45). Evidence depicting a similar situation can be derived from the seasonal influenza vaccine which was only $23 \%$ effective in vaccinees that were infected with a subtype that was different from the one included in the vaccine (51). Although information regarding the unknown DENV serotype is limited, a study by Mustafa et al. has proposed the emergence of a DENV5 serotype (48). By contrast, Hesse argues that the aforesaid is highly unlikely because DENV mutation rate is too low to lead to the creation of a new serotype (44).

\section{CYD-TDV Vaccine Safety}

Our overall findings regarding the safety of CYD-TDV vaccine are in agreement with the findings by da Costa et al. (16) in that none of the increased AEs and ARs were statistically significant. Crevat et al. also concluded that CYD-TDV vaccine does not increase the risk of AEs and ARs in children below 2 years (43). However, this study had a small sample size $(N=90)$, short follow-up time (18 months), and it is not clear how allocation concealment was done. All of which might lead to the actual effect of CYD-TDV vaccine being overestimated. Contrary to the aforesaid, revelations from meta-analysis of studies with

TABLE 5 | Main CYD-TDV immunogenicity findings.

\begin{tabular}{|c|c|c|c|c|c|}
\hline $\begin{array}{l}\text { Dengue } \\
\text { serotype }\end{array}$ & $\begin{array}{l}\text { Number of studies } \\
\text { combined }\end{array}$ & $\begin{array}{l}\text { Heterogeneity } \\
\text { (p-value) }\end{array}$ & $\begin{array}{l}\text { Egger's test for publication bias } \\
\text { ( } p \text {-value) }\end{array}$ & $\begin{array}{l}\text { WMD expressed as GMTs } \\
(95 \% \mathrm{Cl})\end{array}$ & $p$-Value \\
\hline DENV1 & 6 & $93.8 \%(<0.001)$ & 0.01 & 107.5 (70.1-144.9) & $<0.001$ \\
\hline DENV $1^{\mathrm{a}}$ & 5 & $11 \%(0.34)$ & 0.11 & $144.1(117.9-170.2)$ & $<0.001$ \\
\hline DENV2 & 6 & $95.4 \%(<0.001)$ & 0.007 & $176.9(115.4-238.4)$ & $<0.001$ \\
\hline DENV2 ${ }^{\mathrm{a}}$ & 3 & $14.9 \%(0.31)$ & 0.53 & 323.1 (247-398.7) & $<0.001$ \\
\hline DENV3 & 6 & $95.2 \%(<0.001)$ & 0.013 & 221.9 (152.6-291.2) & $<0.001$ \\
\hline DENV3 ${ }^{a}$ & 3 & $41.6 \%(0.18)$ & 0.5 & 439.7 (331.7-547.7) & $<0.001$ \\
\hline DENV4 & 6 & $94.4 \%(<0.001)$ & 0.04 & 152.9 (110.6-195.3) & $<0.001$ \\
\hline DENV4 ${ }^{a}$ & 3 & $37.4 \%(0.2)$ & 0.65 & $105.7(88.7-122.8)$ & $<0.001$ \\
\hline
\end{tabular}

WMD, weighted mean difference expressed as GMTs; GMTs, geometric mean titers (1/dil); DENV 1-4, dengue virus serotypes.

${ }^{a}$ All parameters were determined after resolving heterogeneity by excluding divergent studies. 
longer follow-up time indicate that CYD-TDV increases the risk of injection site reactions significantly thus making it difficult to reject the pre-specified hypothesis (Appendix IV, Figure 8 in Supplementary Material). Similarly, post hoc analysis of the data from Capeding et al. (18) has demonstrated that the risk of hospitalization and severe dengue in children aged between 2 and 5 years vaccinated with CYD-TDV was highly significant ( $\mathrm{RR}=7.45: 1.15-313.8, p$-value not provided) (52). However, post hoc meta-analysis of all studies conducted to assess CYDTDV vaccine safety in children is required before a comprehensive conclusion can be made.

\section{CYD-TDV Vaccine Induced Immunogenicity}

Our immunogenicity findings show that GMTs significantly increased in CYD-TDV vaccinated compared to unvaccinated children. Children in Latin America had higher GMT levels compared to those in Asia. Da Costa et al. (16) have reported similar results and evidence from our findings is sufficient to reject the pre-specified null hypothesis. However, there was significant variation in the effect sizes presented in the included studies. Interestingly, reduced heterogeneity was observed when studies with the same age group were combined regardless of study location except for DENV4. By contrast, combining studies with different age groups resulted in significantly increased heterogeneity regardless of study location and, surprisingly, even within the same study. Furthermore, we found that GMT effect sizes increased as the age of study participants increased, which corresponds with the findings by Tran et al. (39). Taken together, our findings clearly demonstrate that age influences CYD-TDV vaccine induced GMT levels. Included studies demonstrated that prior exposure to dengue viruses increased antibody response during subsequent infection. The observed variations in GMT levels among the included studies might be explained by variations in the burden of dengue infection across countries and between the two regions, with Asian countries experiencing a higher burden compared with Latin American countries (53). Although the PRNT is considered the gold standard, discrepancies between laboratories and regions have been reported $(54,55)$. Furthermore, the scientific community has different views regarding this test. Rainwater-Lovett et al. and Thomas et al. have separately reported that PRNT gives varying results based on the test conditions applied $(56,57)$. Endy has described as confusing the fact that PRNT does not give information on whether an individual will be protected from subsequent dengue infection using cross-reactive neutralizing antibodies (58). The former concern might be responsible for the observed variations in CYD-TDV vaccine elicited GMT levels across the included studies, while the later may be more applicable to the reduced vaccine efficacy against DENV2 as stated above. To the contrary, Timiryasova et al. have concluded that the PRNT test is fit for purpose and can "detect and measure dengue serotype-specific neutralizing antibodies in human serum samples with acceptable intra-assay and inter-assay precision, accuracy/dilutability, specificity, and with a lower limit of quantitation of 10 " (59).

\section{Strengths and Limitations}

This, to the best of our knowledge, is the first systematic review to assess CYD-TDV vaccine efficacy not only in children exclusively but also using primary studies that were designed specifically to determine vaccine efficacy. Our review has demonstrated that children of varying ages respond to CYD-TDV vaccination differently and gender imbalance in the CYD-TDV group introduces heterogeneity when assessing solicited reactions and injection site reactions. Excluding graphically presented data prevented estimation bias. Since all of the included studies were conducted in children located in Asia and Latin America, the findings cannot be generalized to children of all endemic regions.

\section{Implications for Public Health and Research}

Emergence of novel and virulent dengue serotypes has been proposed. To prevent possible pandemics, there is need to strengthen vector control programs, dengue surveillance, diagnostic capabilities and management, and research through capacity building in endemic settings such as Asia, Latin America, and especially Africa, where dengue infection happens to be neglected $(60,61)$. DENV2 included in the CYD-TDV vaccine needs to be updated to match the currently circulating Asian and Latin American variants. In addition, understanding of dengue neutralizing antibodies by investigating whether they are correlates of protection and what titer thresholds against all four serotypes are optimal for protection is warranted. Therefore, the scientific community needs to quickly come to a consensus regarding the PRNT test. Otherwise, new serological tests capable of being standardized to enable inter laboratory comparability, reproducibility and that can accurately and specifically measure dengue correlates of protection must be developed given that this is crucial for vaccine development. The fact that dengue infection is known to cause DHF/DSS and death in children is an indication that passive immunotherapy using serotype-specific neutralizing monoclonal antibodies that target conserved regions of the E protein might be a viable alternative to vaccination. Passive immunotherapy might significantly benefit younger children who are at higher risk and yet respond poorly to vaccines immunologically. Another reason for considering passive immunotherapy is that higher titers of serotype-specific neutralizing monoclonal antibodies can be administered without enhancing ADE, which is mainly caused by low titer levels of cross-reactive antibodies as previously mentioned. Future vaccine trials should consider employing $\geq 25$ months follow-up time, stratify and provide age specific data to facilitate comprehensive and conclusive analyses. Finally, post hoc analysis can reveal vital vaccine-related safety information missed during the duration of the clinical trial. Therefore, the aforementioned analysis should be considered and encouraged where complete clinical data of participants involved in clinical trials are available.

\section{CONCLUSION}

Overall, CYD-TDV vaccine is effective, but less efficacious against DENV2 in children. CYD-TDV vaccine is immunogenic in children with lower GMT levels observed in younger children 
compared to adolescents. Although the vaccine increased the risk of some safety parameters in vaccinated children insignificantly, meta-analysis of studies with long follow-up time revealed that CYD-TDV vaccine significantly increased the risk of solicited injection site reactions. Therefore, post hoc meta-analysis of the long term follow-up data ( $\geq 25$ months) collected from the children previously vaccinated with CYD-TDV are needed before a comprehensive conclusion regarding CYD-TDV vaccine safety in children can be made. However, given the urgency for a dengue vaccine in endemic regions, CYD-TDV should be considered for use in regions where DENV2 is not endemic as currently there is no specific treatment for dengue infection.

\section{AUTHOR CONTRIBUTIONS}

MM designed the study, performed the statistical analysis, and wrote the final report. SK, NT, and GM independently performed searches, selection, and data extraction of published articles. AR resolved disagreements from searches, selection, and data extraction. MB resolved disagreements from searches, selection, and

\section{REFERENCES}

1. Ramakrishnan L, Radhakrishna Pillai M, Nair RR. Dengue vaccine development: strategies and challenges. Viral Immunol (2015) 28(2):76-84. doi:10.1089/vim.2014.0093

2. Halstead SB. Dengue in the Americas and Southeast Asia: do they differ? Rev Panam Salud Publica (2006) 20(6):407-15. doi:10.1590/ S1020-49892006001100007

3. Yacoub S, Mongkolsapaya J, Screaton G. The pathogenesis of dengue. Curr Opin Infect Dis (2013) 26(3):284-9. doi:10.1097/QCO.0b013e32835fb938

4. Brady OJ, Gething PW, Bhatt S, Messina JP, Brownstein JS, Hoen AG, et al. Refining the global spatial limits of dengue virus transmission by evidencebased consensus. PLoS Negl Trop Dis (2012) 6(8):e1760. doi:10.1371/journal. pntd.0001760

5. Bhatt S, Gething PW, Brady OJ, Messina JP, Farlow AW, Moyes CL, et al. The global distribution and burden of dengue. Nature (2013) 496(7446):504-7. doi:10.1038/nature 12060

6. Murrell S, Wu SC, Butler M. Review of dengue virus and the development of a vaccine. Biotechnol Adv (2011) 29(2):239-47. doi:10.1016/j. biotechadv.2010.11.008

7. Guy B, Guirakhoo F, Barban V, Higgs S, Monath TP, Lang J. Preclinical and clinical development of YFV 17D-based chimeric vaccines against dengue, West Nile and Japanese encephalitis viruses. Vaccine (2010) 28(3):632-49. doi:10.1016/j.vaccine.2009.09.098

8. World Health Organization. Global Strategy for Dengue Prevention and Control 2012-2020. (2012). Available from: http://apps.who.int/iris/bitstr eam/10665/75303/1/9789241504034_eng.pdf

9. Villar L, Dayan GH, Arredondo-García JL, Rivera DM, Cunha R, Deseda C, et al. Efficacy of a tetravalent dengue vaccine in children in Latin America. N Engl J Med (2015) 372:113-23. doi:10.1056/NEJMoa1411037

10. WHO. Dengue and Severe Dengue. (2015). Available from: http://www.who. int/mediacentre/factsheets/fs117/en/

11. Screaton G, Mongkolsapaya J, Yacoub S, Roberts C. New insights into the immunopathology and control of dengue virus infection. Nat Rev Immunol (2015) 15(12):745-59. doi:10.1038/nri3916

12. Kurane I. Dengue hemorrhagic fever with special emphasis on immunopathogenesis. Comp Immunol Microbiol Infect Dis (2007) 30(5-6):329-40. doi:10.1016/j.cimid.2007.05.010

13. Faheem M, Raheel U, Riaz MN, Kanwal N, Javed F, Us Sahar Sadaf Zaidi N, et al. A molecular evaluation of dengue virus pathogenesis and its latest vaccine strategies. Mol Biol Rep (2011) 38(6):3731-40. doi:10.1007/s11033-010-0488-1 data extraction. All authors made contributions and reviewed the final report.

\section{FUNDING}

Sincere gratitude goes to William Spence and Hilda Emengo from the Institute of Health and Wellbeing, University of Glasgow and Healthcare Improvement Scotland, respectively, for agreeing to supervise this work. Further gratitude goes to the Chevening Secretariat (Foreign and Commonwealth Office, United Kingdom) for its financial support that led to the realization of this work. SK and AR were supported by RSF grant 15-14-00016 and Program of Competitive Growth of Kazan Federal University. RA was also supported by state assignment 20.5175.2017/6.7 of the Ministry of Education and Science of Russian Federation.

\section{SUPPLEMENTARY MATERIAL}

The Supplementary Material for this article can be found online at http://journal.frontiersin.org/article/10.3389/fimmu.2017.00863/ full\#supplementary-material.

14. Tan GK, Alonso S. Pathogenesis and prevention of dengue virus infection: state-of-the-art. Curr Opin Infect Dis (2009) 22(3):302-8. doi:10.1097/ QCO.0b013e328329ae32

15. Yauch LE, Shresta S. Dengue virus vaccine development. Adv Virus Res (2014) 88:315-72.

16. da Costa VG, Marques-Silva AC, Floriano VG, Moreli ML. Safety, immunogenicity and efficacy of a recombinant tetravalent dengue vaccine: a metaanalysis of randomized trials. Vaccine (2014) 32(39):4885-92. doi:10.1016/j. vaccine.2014.07.008

17. Sabchareon A, Wallace D, Sirivichayakul C, Limkittikul K, Chanthavanich P, Suvannadabba S, et al. Protective efficacy of the recombinant, live-attenuated, CYD tetravalent dengue vaccine in Thai schoolchildren: a randomised, controlled phase 2b trial. Lancet (2012) 380(9853):1559-67. doi:10.1016/S0140-6736(12)61428-7

18. Capeding MR, Tran NH, Hadinegoro SRS, Ismail HIHM, Chotpitayasunondh T, Chua $\mathrm{MN}$, et al. Clinical efficacy and safety of a novel tetravalent dengue vaccine in healthy children in Asia: a phase 3, randomised, observer-masked, placebo-controlled trial. Lancet (2014) 384(9951):1358-65. doi:10.1016/ S0140-6736(14)61060-6

19. Higgins JPT, Altman DG, Gøtzsche PC, Jüni P, Moher D, Oxman AD, et al. The Cochrane Collaboration's tool for assessing risk of bias in randomised trials. BMJ (2011) 343:d5928-5928. doi:10.1136/bmj.d5928

20. Moher D, Liberati A, Tetzlaff J, Altman DG. Reprint - preferred reporting items for systematic reviews and meta-analyses: the PRISMA statement. Phys Ther (2009) 89(9):873-80. doi:10.1136/bmj.b2535

21. United Nations. Convention on the Rights of the Child. (1989). p. 1-23. Available from: http://www.hrweb.org/legal/child.html

22. Hombach J. Guidelines for clinical trials of dengue vaccine in endemic areas. J Clin Virol (2009) 46(Suppl 2):S7-9. doi:10.1016/S1386-6532(09)70287-2

23. World Health Organization. Glossary of terms used in pharmacovigilance. WHO Program Int Drug Monit. (2013). p. 1-6. Available from: http://pvtoolkit. org/toolkit/readers/glossary_of_terms.pdf

24. World Health Organization. Guidelines for Plaque Reduction Neutralization Testing of Human Antibodies to Dengue Viruses. Geneva: WHO (2007). 26 p.

25. Long L. Routine piloting in systematic reviews - a modified approach? Syst Rev (2014) 3:77. doi:10.1186/2046-4053-3-77

26. Bonita R, Beaglehole R, Kjellström T. Basic Epidemiology. 2nd ed. Geneva: World Health Organization (2006). Available from: http://apps.who.int/iris/ bitstream/10665/43541/1/9241547073_eng.pdf

27. Higgins J, Green S. In: Julian PT Higgins and Sally Green, editor. Cochrane Handbook for Systematic Reviews of Interventions. West Sussex: JohnWiley \& Sons Ltd (2011). 
28. Huedo-Medina TB, Sánchez-Meca J, Marín-Martínez F, Botella J. Assessing heterogeneity in meta-analysis: Q statistic or I2 index? Psychol Methods (2006) 11(2):193-206. doi:10.1037/1082-989X.11.2.193

29. Borenstein M, Hedges LV, Higgins J, Rothstein HR. Fixed-effect versus random-effects models. Introduction to Meta-Analysis. West Sussex: John Wiley \& Sons Ltd (2009). p. 77-85.

30. Riedmann EM. Human vaccines \& immunotherapeutics: news. Hum Vaccin Immunother (2013) 9(10):2034-7. doi:10.4161/hv.26189

31. Morrison D, Capeding MR, Poo JL, Forrat R, Zambrano B, Wartel-Tram A, et al. Safety and infectivity of tetravalent Chimeric live attenuated dengue vaccine in different age populations in endemic and non-endemic areas. Am J Trop Med Hyg (2007) 77(5S):99.

32. Crevat D, Reynolds D, Langevin E, Capeding MR. Safety and immunogenicity of a tetravalent dengue vaccine in flavivirus-naive and -immune pediatric populations with two vaccination regimens. Am J Trop Med Hyg (2009) 81(5):113.

33. Bouckenooghe A, Capeding MR, Morrison DN, Poo JL, Lang J, Chambonneau L, et al. Safety and immunogenicity in children and adults from endemic countries and adults from nonendemic countries of a tetravalent, live attenuated dengue vaccine. Am J Trop Med Hyg (2008) 79(6):114.

34. Sabchareon A, Lang J, Chanthavanich P, Yoksan S, Forrat R, Attanath P, et al. Safety and immunogenicity of a three dose regimen of two tetravalent liveattenuated dengue vaccines in five- to twelve-year-old Thai children. Pediatr Infect Dis J (2004) 23(2):99-109. doi:10.1097/01.inf.0000109289.55856.27

35. Poo J, Galan F, Forrat R, Zambrano B, Lang J, Dayan GH, et al. Liveattenuated tetravalent dengue vaccine in dengue-naive children, adolescents, and adults in Mexico City: randomized controlled phase 1 trial of safety and immunogenicity. Pediatr Infect Dis J (2011) 30(1):e9-17. doi:10.1097/ INF.0b013e3181fe05af

36. Capeding RZ, Luna IA, Bomasang E, Lupisan S, Lang J, Forrat R, et al. Liveattenuated, tetravalent dengue vaccine in children, adolescents and adults in a dengue endemic country: randomized controlled phase I trial in the Philippines. Vaccine (2011) 29(22):3863-72. doi:10.1016/j.vaccine.2011.03.057

37. Dayan GH, Garbes P, Noriega F, De Sadovsky ADI, Rodrigues PM, Giuberti C, et al. Immunogenicity and safety of a recombinant tetravalent dengue vaccine in children and adolescents ages 9-16 years in Brazil. Am J Trop Med Hyg (2013) 89(6):1058-65. doi:10.4269/ajtmh.13-0304

38. Leo YS, Wilder-Smith A, Archuleta S, Shek LP, Chong CY, Leong HN, et al. Immunogenicity and safety of recombinant tetravalent dengue vaccine (CYD-TDV) in individuals aged 2-45: phase II randomized controlled trial in Singapore. Hum Vaccines Immunother (2012) 8(9):1259-71. doi:10.4161/ hv. 21224

39. Tran NH, Luong CQ, Vu TQH, Forrat R, Lang J, Vu QD, et al. Safety and immunogenicity of recombinant, live attenuated tetravalent dengue vaccine (CYD-TDV) in healthy Vietnamese adults and children. J Vaccines Vaccin (2012) 3(7):1-8. doi:10.4172/2157-7560.1000162

40. Amar-Singh HSS, Koh MT, Tan KK, Chand LG, Zhou L, Bouckenooghe A, et al. Safety and immunogenicity of a tetravalent dengue vaccine in healthy children aged 2-11 years in Malaysia: a randomized, placebo-controlled, phase III study. Vaccine (2013) 31(49):5814-21. doi:10.1016/j.vaccine.2013.10.013

41. Villar LA, Rivera-Medina DM, Arredondo-García JL, Boaz M, Starr-Spires L, Thakur M, et al. Safety and immunogenicity of a recombinant tetravalent dengue vaccine in 9-16 year olds a randomized, controlled, phase II trial in Latin America. Pediatr Infect Dis J (2013) 32(10):1102-9. doi:10.1097/ INF.0b013e31829b8022

42. Lanata CF, Andrade T, Gil AI, Terrones C, Valladolid O, Zambrano B, et al. Immunogenicity and safety of tetravalent dengue vaccine in 2-11 year-olds previously vaccinated against yellow fever: randomized, controlled, phase II study in Piura, Peru. Vaccine (2012) 30(41):5935-41. doi:10.1016/j. vaccine.2012.07.043

43. Crevat D, Brion JD, Gailhardou S, Laot TM, Capeding MR. First experience of concomitant vaccination against dengue and MMR in toddlers. Pediatr Infect Dis J (2015) 34(8):884-92. doi:10.1097/INF.0000000000000752

44. Hesse RR. Dengue virus evolution and virulence models. Clin Infect Dis (2007) 44(11):1462-6. doi:10.1086/517587
45. Moi ML, Takasaki T, Kurane I. Human antibody response to dengue virus: implications for dengue vaccine design. Trop Med Health (2016) 44(1):1. doi:10.1186/s41182-016-0004-y

46. Thomas SJ, Rothman AL. Trials and tribulations on the path to developing a dengue vaccine. Am J Prev Med (2015) 49(6):S334-44. doi:10.1016/j. amepre.2015.09.006

47. Haven N. Molecular evolution of dengue type 2 virus in Thailand. Trop Med (1998) 58(1):96-101.

48. Mustafa MS, Rasotgi V, Jain S, Gupta V. Discovery of fifth serotype of dengue virus (denv-5): a new public health dilemma in dengue control. Med J Armed Forces India (2015) 71(1):67-70. doi:10.1016/j.mjafi.2014.09.011

49. Malavige GN, Fernando S, Fernando DJ, Seneviratne SL. Dengue viral infections. Postgrad Med J (2004) 80(948):588-601. doi:10.1136/pgmj.2004.019638

50. Wahala MPB, Donaldson EF, de Alwis R, Accavitti-Loper MA, Baric RS, de Silva AM. Natural strain variation and antibody neutralization of dengue serotype 3 viruses. PLoS Pathog (2010) 6(3):e1000821. doi:10.1371/journal. ppat.1000821

51. World Health Organization. Questions and Answers: Vaccine Effectiveness Estimates for Seasonal Influenza Vaccines. (2015). p. 1-4. Available from: http://www.who.int/influenza/vaccines/virus/recommendations/201502_ qanda_vaccineeffectiveness.pdf

52. World Health Organization. Safety of CYD-TDV Dengue Vaccine: Weekly Epidemiological Record. Geneva: WHO (2016). p. 421-8. Available from: http://www.who.int/vaccine_safety/committee/reports/wer9034.pdf

53. L'Azou M, Moureau A, Sarti E, Nealon J, Zambrano B, Wartel TA, et al. Symptomatic dengue in children in 10 Asian and Latin American countries. N Engl J Med (2016) 374(12):1155-66. doi:10.1056/NEJMoa1503877

54. World Health Organization. Dengue: guidelines for diagnosis, treatment, prevention and control. Prev Control (2009) 409(3):160.

55. Tang KF, Ooi EE. Diagnosis of dengue: an update. Expert Rev Anti Infect Ther (2012) 10(8):895-907. doi:10.1586/eri.12.76

56. Thomas SJ, Nisalak A, Anderson KB, Libraty DH, Kalayanarooj S, Vaughn DW, et al. Dengue plaque reduction neutralization test (PRNT) in primary and secondary dengue virus infections: how alterations in assay conditions impact performance. Am J Trop Med Hyg (2009) 81(5):825-33. doi:10.4269/ajtmh.2009.08-0625

57. Rainwater-Lovett K, Rodriguez-Barraquer I, Cummings DA, Lessler J. Variation in dengue virus plaque reduction neutralization testing: systematic review and pooled analysis. BMC Infect Dis (2012) 12(1):233. doi:10.1186/ 1471-2334-12-233

58. Endy TP. Human immune responses to dengue virus infection: lessons learned from prospective cohort studies. Front Immunol (2014) 5:183. doi:10.3389/ fimmu.2014.00183

59. Timiryasova TM, Bonaparte MI, Luo P, Zedar R, Hu BT, Hildreth SW. Optimization and validation of a plaque reduction neutralization test for the detection of neutralizing antibodies to four serotypes of dengue virus used in support of dengue vaccine development. Am J Trop Med Hyg (2013) 88(5):962-70. doi:10.4269/ajtmh.12-0461

60. Lam SK. Challenges in reducing dengue burden; diagnostics, control measures and vaccines. Expert Rev Vaccines (2013) 12(9):995-1010. doi:10.1586/ 14760584.2013.824712

61. Amarasinghe A, Kuritsk JN, Letson GW, Margolis HS. Dengue virus infection in Africa. Emerg Infect Dis (2011) 17(8):1349-54. doi:10.3201/eid1708.101515

Conflict of Interest Statement: The authors declare that the research was conducted in the absence of any commercial or financial relationships that could be construed as a potential conflict of interest.

Copyright (c) 2017 Malisheni, Khaiboullina, Rizvanov, Takah, Murewanhema and Bates. This is an open-access article distributed under the terms of the Creative Commons Attribution License (CC BY). The use, distribution or reproduction in other forums is permitted, provided the original author(s) or licensor are credited and that the original publication in this journal is cited, in accordance with accepted academic practice. No use, distribution or reproduction is permitted which does not comply with these terms. 